\title{
Relationship between Ionic Mobility and Segmental Mobility in Polymers in the Liquid State
}

\author{
Hiroyuki SASABe and Shogo Saito \\ Materials Division, Electrotechnical Laboratory, Tanashi, Tokyo, Japan.
}

(Received February 3, 1972)

\begin{abstract}
Temperature and pressure dependences of the ionic d.c. conductivity $\sigma$ $(\propto \sigma)$ and the segmental mobility $\left(\propto \tau^{-1}\right)$ were analysed in terms of the WLF and the Ferry-Stratton(FS) equations, respectively, where $\tau$ is the dielectric relaxation time for the segmental motion above the glass transition. The WLF parameter $C_{2}$ for $\sigma$ is nearly equal to that for $\tau^{-1}$, and the FS parameter $b_{2}$ for $\sigma$ is also nearly equal to that for $\tau^{-1}$ in some cases. In the other cases, however, this does not hold. If $\tau^{-1}$ and the ionic mobility $\mu$ are assumed to be described by the same $C_{2}$, the former cases correspond to a constant carrier density and the latter to variable carrier density. In the case of constant carrier density, the relation $\sigma(T, P)[\tau(T, P)]^{m}=$ const. is derived from experimental results. This is designated as a "modified Walden's rule." The exponent $m$ is given by the ratio $C_{1}(\sigma) / C_{1}\left(\tau^{-1}\right)$ or $b_{1}(\sigma) / b_{1}\left(\tau^{-1}\right)$. The physical meaning of $m$ is the ratio of critical hole size for ionic charge transport to that for the segmental motion.

KEY WORDS D.C. Conductivity / Segmental Mobility / Walden's Rule / WLF Equation / Ferry Stration Equation / Critical Volume /
\end{abstract}

In previous papers ${ }^{1,2}$ it has been found that the temperature coefficient of d.c. conductivity in a polymer changes suddenly at the glass transition temperature $T_{\mathrm{g}}$ and is much larger in the liquid state than in the glassy state. This phenomenon may be interpreted qualitatively in such a way that the segmental motion of amorphous polymer chains favors a remarkable increase in the mobility of current carriers. Temperature and pressure dependences of the d.c. conductivity $\sigma$ have been analyzed in terms of the theory of the activation process; temperature dependence of $\sigma$ was assumed to be of the Arrhenius-type. However if one pays attention to a change of $\sigma$ with temperature, it will be noticed that the change follows the Williams-Landel-Ferry(WLF) equation rather than the Arrhenius equation. Temperature dependence of the dielectric relaxation time $\tau$ for the micro-Brownian motion of amorphous polymer chains which is related to the segmental mobility is also represented by the WLF equation. Pressure dependences of $\tau$ and $\sigma$ are both represented by the Ferry-Stratton equation but their parameters are not identical. Therefore, some relationship between the molecular motion of polymers related to the dielectric relaxation and that of the transport of ions as current carriers is expected. For poly (vinyl chloride) (PVC), we have determined the d.c. conductivity $\sigma$ and the average segmental mobility as a function of temperature and pressure in the liquid state. It has been also indicated that ionic conduction is the predominant mechanism of the d.c. conduction in PVC. Therefore it is possible to find quantitatively the effect of segmental motion on the d.c. (ionic) conduction behavior. The purpose of this paper is to find the relationship between the ionic conduction and the segmental mobility in polymers.

Now, it will be recalled that the relation for a simple liquid is given by,

$$
\sigma(T) \eta(T)=C
$$

which is well known as Walden's rule ${ }^{3}$. Here $C$ is a temperature-independent constant and $\eta(T)$ is the viscosity. This equation is a good approximation in describing the relationship between d.c. conductivity and viscosity in low-molecularweight liquids. As for a polymer in the liquid state, the relaxation time for the mechanical $\alpha_{\mathrm{a}}$ relaxation process due to the segmental motion of polymer chains is closely connected to the viscosity $\eta$; that is, the relaxation time is pro- 
portional to the local viscosity. As the dielectric $\alpha_{\mathrm{a}}$ relaxation is attributed to the micro-Brownian motion of polymer segments and reflects the same molecular motion as the mechanical $\alpha_{\mathrm{a}}$ relaxation process, the dielectric relaxation time for the $\alpha_{\mathrm{a}}$ process is also proportional to the viscosity. This concept is also confirmed in Figure 1. That is, Figure 1 shows the similarity between the temperature dependence of

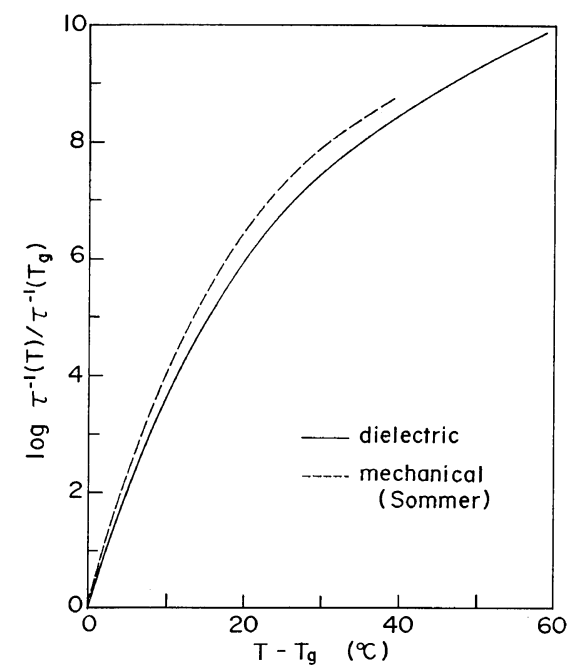

Figure 1. Comparison of temperature dependence of dielectric relaxation time with that of mechanical relaxation time (by Sommer).

mechanical relaxation time by Sommer ${ }^{4}$ and the dielectric relaxation time $\tau$ for PVC. Hence a similar relationship to the Walden's rule would be expected between the conductivity $\sigma$ or the carrier mobility $\mu$ in a polymer above the glass transition temperature and the relaxation time $\tau$ for the $\alpha_{\mathrm{a}}$ relaxation process.

\section{DISCUSSION}

\section{Relationship between $\sigma$ and $\tau$ as a Function of Temperature}

Samples used in the present study were poly (vinyl chloride) (PVC), plasticized PVC (pl-PVC; $\mathrm{PVC}+$ tetralin (23 wt $\%$ )), poly (vinylidene chloride) (PVDC), poly(vinyl acetate) (PVAc), amorphous poly (ethylene terephthalate) (amPET), and polycarbonate (PC). The d.c. conductivity $\sigma$ and the average dielectric relaxation time $\tau$ for the $\alpha_{\mathrm{a}}$ relaxation in each sample were measured at various temperatures above $T_{\mathrm{g}}$. The devices for dielectric and d.c. measure-

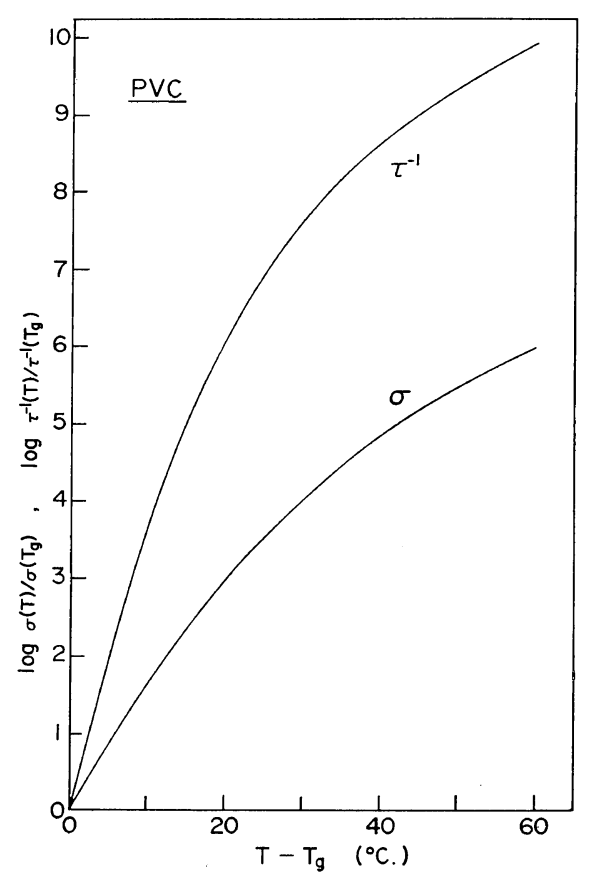

Figure 2. Temperature dependence of d.c. conductivity and segmental mobility for PVC.

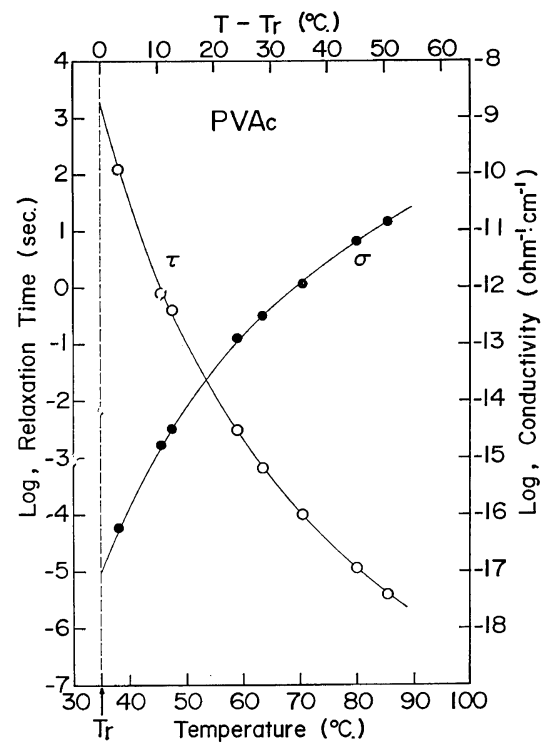

Figure 3. Temperature dependence of d.c. conductivity and segmental mobility for PVAc. 
ments as functions of temperature and pressure have been described in detail in ref 1 . The segmental mobility is assumed to be inversely proportional to $\tau$. Figures 2 and 3 show the temperature dependence of $\sigma$ and $\tau^{-1}\left(\propto f_{\mathrm{m}}\right)$ in PVC and PVAc, respectively, for example. The curves can be fitted to the WLF-type equation:

$$
\log \tau^{-1}(T) / \tau^{-1}\left(T_{\mathrm{g}}\right)=C_{1}\left(T-T_{\mathrm{g}}\right) /\left[C_{2}+\left(T-T_{\mathrm{g}}\right)\right] \text { (2) }
$$

and

$$
\log \sigma(T) / \sigma\left(T_{\mathrm{g}}\right)=C_{1}{ }^{\prime}\left(T-T_{\mathrm{g}}\right) /\left[C_{2}{ }^{\prime}+\left(T-T_{\mathrm{g}}\right)\right]
$$

The parameters $C_{1}, C_{2}, C_{1}{ }^{\prime}$, and $C_{2}{ }^{\prime}$ for various samples are listed in Table I.

Now let's consider the ratio of $\log \sigma(T) / \log \sigma$ $\left(T_{\mathrm{g}}\right)$ to $\log \tau^{-1}(T) / \tau^{-1}\left(T_{\mathrm{g}}\right)$. Figure 4 shows the ratio vs. $1 / T$ plots for various samples. There are two groups; that is,
Group A...The ratio is dependent on $T$

...PVC, PMMA, am-PET, and PC

Group B...The ratio is independent of $T$ ...pl-PVC, PVAc, and PVDC

In the case of group $\mathrm{B}$, the relations $C_{1}{ }^{\prime} \neq$ $C_{1}$ and $C_{2}{ }^{\prime} \simeq C_{2}$ are obtained for each sample. On the other hand, the relations $C_{1}{ }^{\prime} \neq C_{1}$ and $C_{2}{ }^{\prime} \neq C_{2}$ are obtained for samples belonging to group $\mathrm{A}$. In the former case the ratio $[\log \sigma$ $\left.(T) / \sigma\left(T_{\mathrm{g}}\right)\right] /\left[\log \tau^{-1}(T) / \tau^{-1}\left(T_{\mathrm{g}}\right)\right] \quad(\equiv m)$ is given by the ratio of $C_{1}{ }^{\prime}$ to $C_{1}$; that is,

$$
m=C_{1}{ }^{\prime} / C_{1}
$$

As shown in Table I, $m$ is very close to $C_{1}{ }^{\prime} / C_{1}$ for each sample belonging to group $B$. This evidence leads to the relation

$$
\sigma(T)[\tau(T)]^{m}=\text { const. }
$$

for group B.

Table I. WLF parameters for various samples

\begin{tabular}{lrrrrrrrr}
\hline Polymer & $T_{\mathrm{g}},{ }^{\circ} \mathrm{C}$ & $C_{1}$ & \multicolumn{1}{c}{$C_{2}$} & \multicolumn{1}{c}{$C_{1}{ }^{\prime}$} & $C_{2}{ }^{\prime}$ & $m$ & $C_{1}{ }^{\prime} / C_{1}$ & $\begin{array}{c}\Delta H, \\
\mathrm{kcal} / \mathrm{mol}\end{array}$ \\
\hline pl-PVC & 30 & 11.81 & 29.3 & 8.54 & 34.5 & 0.66 & 0.72 & \\
PVAc & 31 & 15.58 & 41.8 & 11.18 & 42.2 & 0.71 & 0.72 & \\
PVDC & -10 & 11.86 & 109.7 & 11.87 & 114.0 & 1.01 & 1.00 & \\
\hline PVC & 76 & 16.40 & 35.7 & 12.50 & 67.1 & $(0.25)$ & - & 84 \\
PMMA & 85 & 22.23 & 48.3 & 8.73 & 67.8 & $(0.22)$ & - & 122 \\
am-PET & 70 & 11.98 & 42.0 & $\overline{6.12}$ & -55.6 & $(0.25)$ & - & 64 \\
PC & 140 & 11.87 & 37.2 & $6.18)$ & - & 46 \\
\hline
\end{tabular}

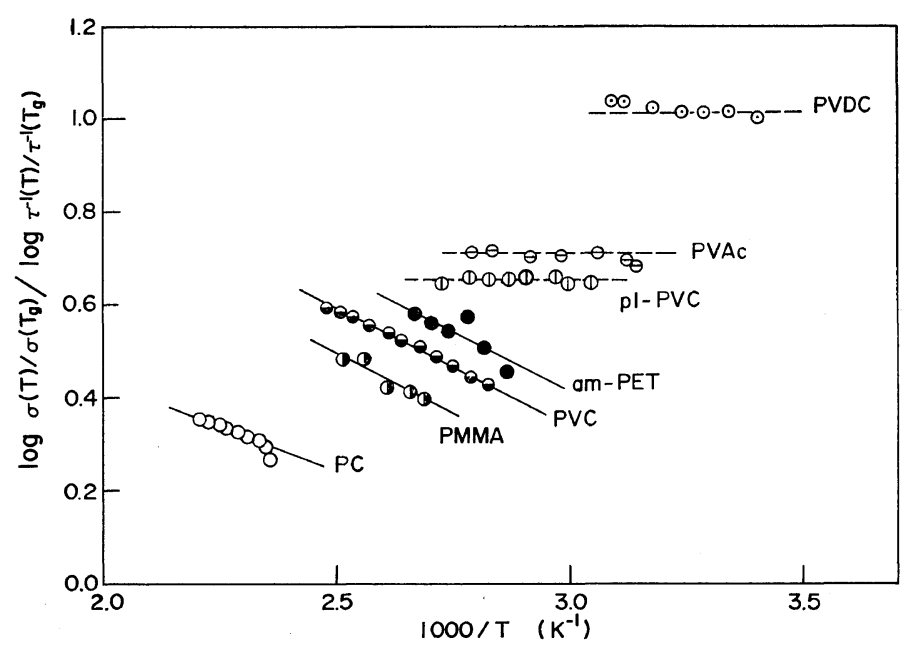

Figure 4. Relation between $\left[\log \sigma(T) / \sigma\left(T_{\mathrm{g}}\right)\right] /\left[\log \tau^{-1}(\boldsymbol{T}) / \tau^{-1}\left(\boldsymbol{T}_{\mathrm{g}}\right)\right]$ and $1 / T$ for various samples. 
Relationship between Ionic and Segmental Mobilities

Relationship between $\sigma$ and $\tau$ at a Constant Temperature as a Function of Pressure

Figures 5 and 6 show the effect of pressure on $\tau$ and $\sigma$ at a fixed temperature for PVC $\left(107^{\circ} \mathrm{C}\right)$ and for PVAc $\left(88^{\circ} \mathrm{C}\right)$, respectively. The curves are fitted to the Ferry-Stratton equation:

$$
\log \tau^{-1}(P) / \tau^{-1}(0)=-b_{1} P /\left(b_{2}-P\right)
$$

and

$$
\log \sigma(P) / \sigma(0)=-b_{1}{ }^{\prime} P /\left(b_{2}{ }^{\prime}-P\right)
$$

The parameters $b_{1}, b_{2}, b_{1}{ }^{\prime}$, and $b_{2}{ }^{\prime}$ for various samples are listed in Table II.

Figure 7 shows the relation between the ratio $[\log \sigma(P) / \sigma(0)] /\left[\log \tau^{-1}(P) / \tau^{-1}(0)\right]$ and $P$. There

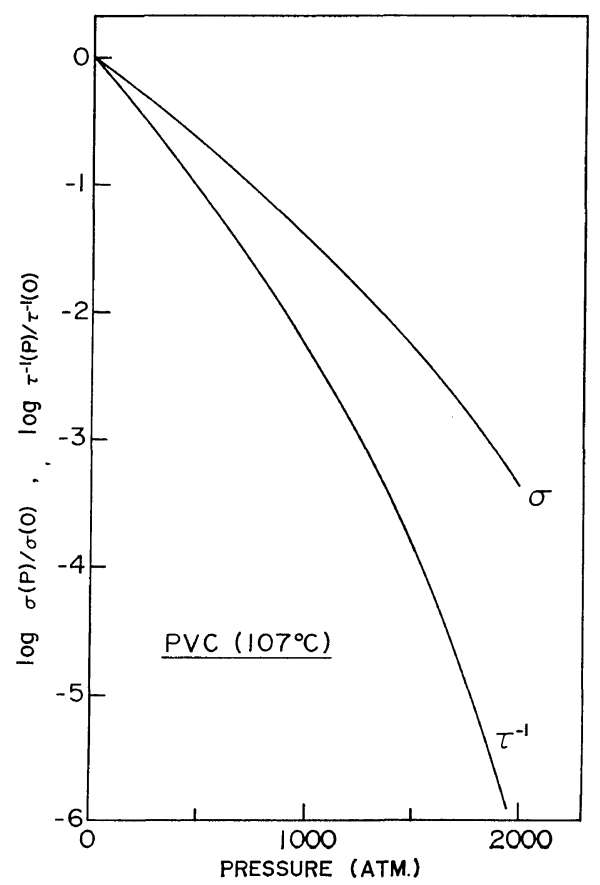

Figure 5. Pressure dependence of d.c. conductivity and segmental movility for PVC at $107^{\circ} \mathrm{C}$.

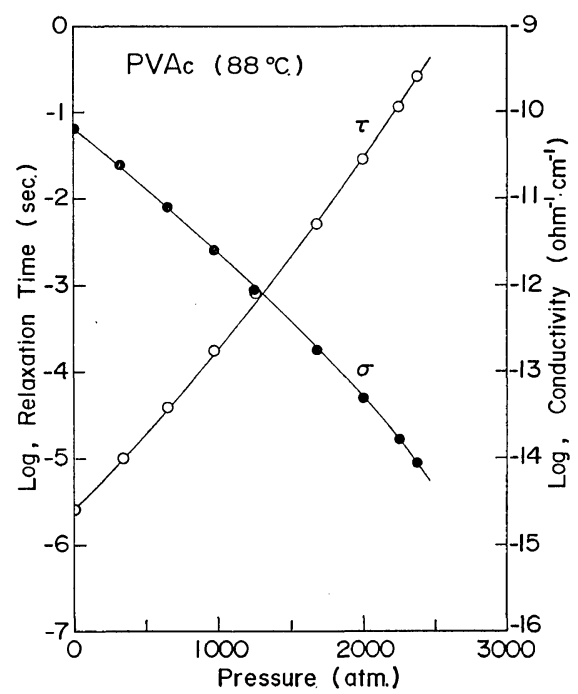

Figure 6. Pressure dependence of d.c. conductivity and segmental mobility for PVAc at $88^{\circ} \mathrm{C}$.

are two groups; that is, Group $\mathrm{A}^{\prime}$...The ratio is dependent on $P$ ...PVC, PMMA

Group $\mathbf{B}^{\prime}$... The ratio is independent of $P$ ...PVAc, pl-PVC

It is very important that the sample belonging to group $\mathbf{A}^{\prime}$ (or $\mathbf{B}^{\prime}$ ) belongs to group $\mathbf{A}$ (or $\mathbf{B}$ ). For samples belonging to group $\mathbf{B}^{\prime}$, the relations $b_{1}{ }^{\prime} \neq b_{1}$ and $b_{2}{ }^{\prime} \simeq b_{2}$ are obtained, whereas for samples belonging to group $\mathrm{A}^{\prime}, b_{1}{ }^{\prime}$, and $b_{2}{ }^{\prime}$ are different from $b_{1}$ and $b_{2}$, respectively. In the case of group $\mathbf{B}^{\prime}$, the ratio $[\log \sigma(P) / \sigma(0)] /$ $\left[\log \tau^{-1}(P) / \tau^{-1}(0)\right]\left(\equiv m^{\prime}\right)$ is given by the ratio of $b_{1}{ }^{\prime}$ to $b_{1}$; that is,

$$
m^{\prime}=b_{1}{ }^{\prime} / b_{1}
$$

This relation is supported for the samples belonging to group $\mathbf{B}^{\prime}$ as shown in Table II, and leads to the following formulation;

$$
\sigma(\boldsymbol{P})[\tau(\boldsymbol{P})]^{m \prime}=\text { const. }
$$

Table II. FS parameters for various samples

\begin{tabular}{lcrrrrrr}
\hline Polymer & $\mathrm{T},{ }^{\circ} \mathrm{C}$ & \multicolumn{1}{c}{$b_{1}$} & $b_{2}$ & $b_{1}{ }^{\prime}$ & $b_{2}{ }^{\prime}$ & $m^{\prime}$ & $b_{1}{ }^{\prime} / b_{1}$ \\
\hline pl-PVC & 94 & 2.41 & 2753 & 1.18 & 2016 & 0.27 & 0.49 \\
PVAc & 88 & 22.22 & 1280 & 15.39 & 1184 & 0.77 & 0.69 \\
\hline PVC & 107 & 9.76 & 5240 & 5.43 & 5560 & $(0.45)$ & - \\
PMMA & 102 & 1.36 & 2540 & 0.88 & 3350 & $(0.57)$ & - \\
am-PET & 86 & 30.40 & 4330 & 11.12 & 4404 & - & - \\
\hline
\end{tabular}




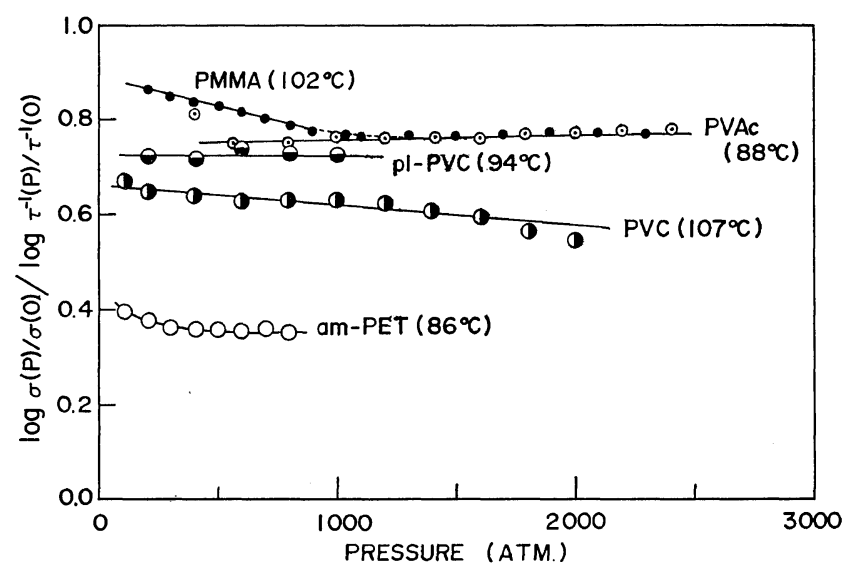

Figure 7. Relation between $[\log \sigma(P) / \sigma(0)] /\left[\log \tau^{-1}(P) / \tau^{-1}(0)\right]$ and pressure for various samples.

Values of $m$ and $m^{\prime}$ for a polymer are nearly equal to each other. Moreover, the physical meaning of them is also identical, which will be discussed later. Therefore eq 4 and 7 can be combined as

$$
\sigma(T, P)[\tau(T, P)]^{m}=\text { const. }
$$

We have designated this relation as a modified Walden's rule.

In the case of group B which has a relatively high conductivity, it might be assumed that the carrier density $n$ is constant. Then eq 8 is rewritten as

$$
\mu(T, P)[\tau(T, P)]^{m}=\text { const. }
$$

If we assume in the case of group $A$ that the carrier mobility $\mu$ follows the modified Walden's rule, eq 9, and the density of carrier created due to thermal ionization is much larger than that contained in original sample, $\sigma$ is expressed in the form

$$
\sigma(T, P)=C \exp \left[-\frac{\Delta H+P \Delta V}{2 R T}\right][\tau(T, P)]^{-m}
$$

where $\Delta H$ is the ionization energy and $\Delta V$ is the volume change associated with ionization. This equation leads to the relations

$\left[\log \sigma(T) / \sigma\left(T_{\mathrm{g}}\right)\right] /\left[\log \tau^{-1}(T) / \tau^{-1}\left(T_{\mathrm{g}}\right)\right]=a-b(1 / T)(11)$ and

$$
[\log \sigma(\boldsymbol{P}) / \sigma(0)] /\left[\log \tau^{-1}(\boldsymbol{P}) / \tau^{-1}(0)\right]=a^{\prime}-b^{\prime} \boldsymbol{P}
$$

These relations are shown in Figures 4 and 7.
Values of $\Delta H$ and $\Delta V$ for each sample belonging to group A can be calculated from intercepts $\left(a, a^{\prime}\right)$ and slopes $\left(b, b^{\prime}\right)$ (Table I). Values of $\Delta H$ are located close to the values of the bond-dissociation energy for $\mathrm{C}-\mathrm{C}(83.1 \mathrm{kcal}$ $\left.\mathrm{mol}^{-1}\right), \quad \mathrm{C}-\mathrm{Cl}\left(78.5 \mathrm{kcal}^{\mathrm{mol}}{ }^{-1}\right), \quad \mathrm{C}-\mathrm{O}(84.0$ $\left.\mathrm{kcal} \mathrm{mol}^{-1}\right)$, etc. ${ }^{5}$

\section{Physical Meaning of $m$}

As has been discussed in the preceeding sections, the exponent $m$ in the modified Walden's rule for group $\mathrm{B}$ is expressed by the parameters $C_{1}, C_{1}{ }^{\prime}, b_{1}$, and $b_{1}{ }^{\prime}$;

$$
m=C_{1}{ }^{\prime} / C_{1}=b_{1}{ }^{\prime} / b_{1}
$$

Therefore, in order to consider the physical meaning of $m$, it is necessary to look into the physical meaning of $C_{1}$ and $b_{1}$.

According to the free volume theory developed by Cohen and Turnbull, ${ }^{6}$ the WLF parameters $C_{1}$ and $C_{2}$ are given by

$$
C_{1}=\gamma v^{*} / 2.3 f_{\mathrm{g}} \bar{v}_{\mathrm{m}} \text { and } \quad C_{2}=f_{\mathrm{g}} / \alpha_{\mathrm{f}}
$$

where $v^{*}$ is the cirtical volume large enough to permit a molecule to jump in after the displacement, $\bar{v}_{\mathrm{m}}$ is the average molecular volume, $\gamma$ is a numerical factor introduced to correct for overlap of free volume, $f_{\mathrm{g}}$ is the free volume fraction at the glass transition temperature, and $\alpha_{\mathrm{f}}$ is the thermal expansion coefficient of the free volume. If it is assumed that $v_{\mathrm{f}}=v_{\mathrm{fg}}+$ $\alpha_{\mathrm{f}} \bar{v}_{\mathrm{m}}\left(T-T_{\mathrm{g}}\right)-\beta_{\mathrm{f}} \bar{v}_{\mathrm{m}} \boldsymbol{P}$, the FS parameters $b_{1}$ and $b_{2}$ are easily determined from Cohen-Turnbull theory as 


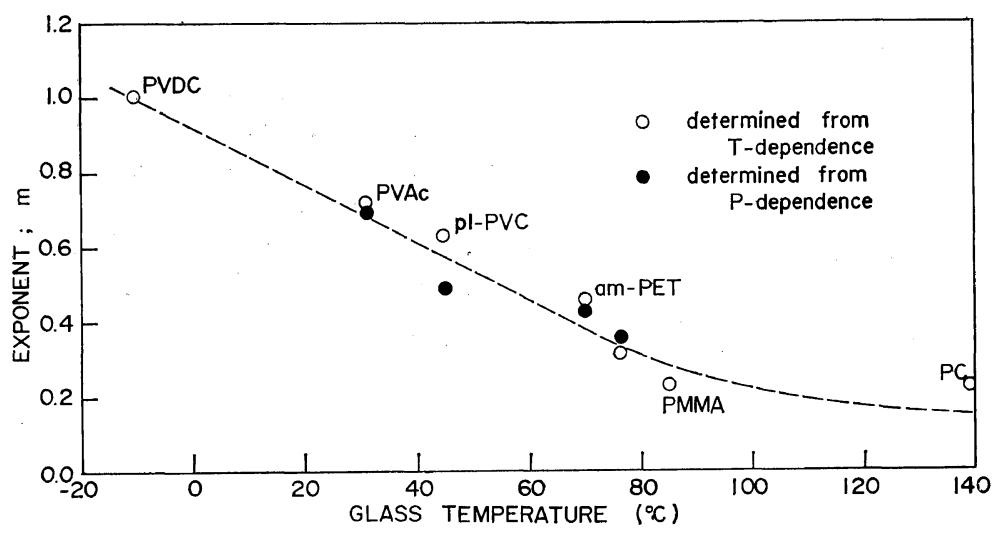

Figure 8. Relation between the exponent $m$ and glass-transition temperature $T_{\mathrm{g}}$ for various samples.

$$
b_{1}=\gamma v^{*} / 2.3 f_{0} \bar{v}_{\mathrm{m}} \text { and } b_{2}=f_{0} / \beta_{\mathrm{f}}
$$

where $v_{\mathrm{fg}}$ is the free volume at $T_{\mathrm{g}}$ under 1 atm., $f_{0}$ is the free volume fraction at atmospheric pressure, and $\beta_{\mathrm{f}}$ is the isothermal compressibility of free volume.

Parameters $C_{2}$ and $b_{2}$ reflect the characteristics of a polymer matrix only. On the other hand, parameters $C_{2}$ and $b_{2}$ reflect not only the characteristics of a polymer matrix but the size of the moving unit. Consequently, the exponent $m$ is regarded as a measure of the size of the ionic charge carriers in comparison to that of moving polymer segments. For low-molecularweight liquids the value of $m$ is equal to unity (Walden's rule). This may be interpreted as the size of the charge carries in a low-molecular-weight liquid is of the same order as that of the molecules of a medium liquid. On the other hand, values of $m$ for various polymers are less than unity. This evidence indicates that the size of moving unit for d.c. conduction is much smaller than that for the segmental motion.

Figure 8 shows the relationship between $T_{\mathrm{g}}$ and $m$ for various polymers. The polymer with a higher $T_{\mathrm{g}}$ has a smaller value of $m$. This tendency is reasonable if we take the following facts into consideration; though $T_{\mathrm{g}}$ of a polymer depends on the chain stiffness and the concentration of plasticizers, the size of monomer units in a polymer with a higher $T_{\mathrm{g}}$ is generally large and the structure of polymer chains is bulky in the case of unplasticized polymers. On the other hand, the size of charge carriers (ions) in polymers is not so different irrespective of the variety of the host polymers. Consequently it is concluded that a polymer with a higher $T_{\mathrm{g}}$ has a larger moving unit and hence it has a smaller value of $m$.

\section{CONCLUSION}

Effect of temperature on d.c. conductivity $\sigma$ was represented by the WLF-type equation with values of $C_{1}^{\prime}$ and $C_{2}{ }^{\prime}$ different from the equation for the dielectric relaxation time $\tau$ for the $\alpha_{\mathrm{a}}$ relaxation. In the case of group $\mathbf{B}$ which has a relatively high conductivity, the parameter $C_{2}{ }^{\prime} \simeq C_{2}$ but $C_{1}{ }^{\prime} \neq C_{1}$. If the carrier density $n$ is assumed to be constant, $\mu \tau^{m}=$ const., then in this case the relation $\sigma(T)[\tau(T)]^{m}$ $=$ const. was confirmed from the experimental results.

Pressure dependences of $\sigma$ and $\tau$ are described by the FS-type equations. In the case of group $\mathrm{B}$, parameters $b_{2}^{\prime}$ and $b_{2}$ are nearly equal but parameters $b_{1}{ }^{\prime}$ and $b_{1}$ differ from each other. The relation $\sigma(P)[\tau(P)]^{m}=$ const. was also obtained from the experimental results. Therefore $\sigma$ and $\tau$ can be related each other as $\sigma(T, P)$ $[\tau(T, P)]^{m}=$ const. This relation is designated as a modified Walden's rule.

The exponent $m$ is found to be determined by the WLF or FS parameters as $m=C_{1}{ }^{\prime} C_{1}=b_{1}{ }^{\prime}$ $b_{1}$ and regarded as a measure of the size of the charge carriers (ions) in comparison with that of moving polymer segments. The fact that 
the value of $m$ is less than unity indicates the size of moving unit for the d.c. conduction is much smaller than that for the segmental motion.

In the case of group $\mathrm{A}$, parameters $C_{1}{ }^{\prime}, C_{2}{ }^{\prime}$, $b_{1}{ }^{\prime}$, and $b_{2}{ }^{\prime}$ for $\sigma$ are different from $C_{1}, C_{2}, b_{1}$, and $b_{2}$ for $\tau$, respectively. If carrier density $n$ is assumed to change with $T$ and $P$ due to thermal ionization and carrier mobility $\mu$ is related to $\tau$ as $\mu \tau^{m}=$ const., the bond-dissociation energy $\Delta H$ can be calculated $\Delta H=50 \sim 120 \mathrm{kcal}$ $\left.\mathrm{mol}^{-1}\right)$.

A polymer with a higher $T_{\mathrm{g}}$ has a smaller value of $m$.

\section{REFERENCES}

1. S. Saito, H. Sasabe, T. Nakajima, and K. Yada, J. Polym. Sci., Part A-2, 6, 1297 (1968).

2. S. Saito, Rep. Progr. Polym. Phys. Japan, 12, 407 (1969).

3. P. Walden, Z. Phys. Chem., 55, 249 (1906).

4. W. Sommer, Kolloid Z., 167, 97 (1959).

5. L. Pauling, in "The Nature of the Chemical Bond," Cornell University Press, New York, N.Y., 1960, p 85.

6. M.H. Cohen and D. Turnbull, J. Chem. Phys., 31, 1164 (1959). 